ren Stellen behandelte Ronald Schill gehört, keine effektvollen Populisten. Verständlicherweise besteht nach wie vor ein Populismus-Tabu, das in dieser Form weder in Italien noch in Österreich vorherrscht. Darüber hinaus wird hierzulande meist von einem Rechtspopulismus ausgegangen, was der Regierungswirklichkeit in der Welt keineswegs entspricht, wo linke beziehungsweise demokratische Populismen vorhanden sind.

Decker geht es in den hier versammelten Beiträgen eigentlich weniger um den Populismus als um eine Analyse politischer Konstellationen und Situationen. Der Untertitel „Beiträge zum Zustand der Demokratie und des Parteiensystems" trifft insofern sein Anliegen besser. Das Spannungsverhältnis zwischen repräsentativen Institutionen und plebiszitären Elementen spielt dabei eine Rolle. In südlichen und östlichen Gesellschaften hat dieser Konflikt in jüngster Zeit weiter zugenommen - wie die Ereignisse in Thailand, der Ukraine oder Venezuela verdeutlichen. Es ist gut möglich, dass in einem künftigen Band aktueller Beiträge von Frank Decker diese Themen eine noch prominentere Rolle gewinnen werden.

Nikolaus Werz

\title{
Das Internet als Innovation oder Stolperstein für Demokratie: eine „netzrealistische“ Erschließung
}

Kneuer, Marianne (Hrsg.): Das Internet: Bereicherung oder Stressfaktor für die Demokratie? (Veröffentlichungen der Deutschen Gesellschaft für Politikwissenschaft, Band 31), Nomos Verlagsgesellschaft, Baden-Baden 2013, 276 Seiten, € 44,-.

Der technische Fortschritt ist ein ständiger Begleiter der Menschheitsgeschichte, doch gerade mit der Erfindung von Personal Computer, Internet und ihren zahlreichen neueren Derivaten ist die Durchdringung des Alltags an einem Punkt angelangt, an dem die Frage „Bereicherung oder Stressfaktor für die Demokratie?“ durchaus berechtigt ist. Mahnende Kritik gibt es indes im Überfluss, man denke nur an den Schlusssatz von Stéphane Hessels Schrift „Empört euch!“: Dort wird gar zum „friedlichen Aufstand gegen den Missbrauch der Massenkommunikationsmittel und der Verführung unserer Jugend zum Massenkonsum"1 aufgerufen.

Eine unaufgeregte „netzrealistische“ Erschließung der oben genannten Fragestellung bietet der von Marianne Kneuer herausgegebene Band. Die Publikation geht auf die Jahrestagung der Deutschen Gesellschaft für Politikwissenschaft (DGfP) im November 2012 an der Universität Hildesheim zurück. Ihr Ziel ist es, das „Potenzial neuer Nutzungsformen digitaler Medien im Spannungsfeld zwischen die Demokratie bereichernden oder -belastenden Wirkungen abzutasten und die Implikationen aufzuzeigen“ (Marianne Kneuer, S. 8 f.). Zwölf Autoren steuern elf Beiträge zu diesem Vorhaben bei.

Auf die Einleitung folgen drei Teile, die sich mit der Bedeutung des Internets für: 1. Transparenz, Repräsentation und Legitimation, 2. Partizipation und 3. Demokratisierung von Autokratien beschäftigen. Kurz gefasst könnte man von einer theoretischen und einer doppelten 
empirischen Vermessung des Forschungsfeldes - in den westlichen Demokratien und darüber hinaus - sprechen. Dabei ermöglicht die Bewertung als „Bereicherung“ oder „Belastung“ für die Demokratie, das breite Themenfeld auf einen gemeinsamen Nenner zu bringen.

Der erste Schwerpunkt umfasst vier Beiträge. Der Aufsatz von Barbara Zehnpfennig „Mehr Transparenz - weniger Demokratie?“ beschäftigt sich mit der Veränderung der Öffentlichkeit als Raum (S. 42) gegenüber dem Privaten (S. 44 - 46). Die Feststellung, dass das Internet im Besonderen durch „Strukturlosigkeit“ (S. 43) gekennzeichnet ist und eine Grenze zwischen „Virtualität und Realität“ (S. 49) besteht, deutet auf eine differenzierte Beantwortung der übergeordneten Fragestellung. Auf die Frage von Transparenz und Verwendung von Informationen nehmen James W. Davis und Miriam Meckel in ihrem Beitrag „Political Power and the Requirements of Accountability in the Age of Wikileaks" ebenfalls Bezug. Dem in Deutschland sehr prominenten Thema „Urheberrecht, Selbstbestimmung und Subjekt“ gewinnt Michael Hassemer eine interessante Seite ab, wenn er resümiert, dass „in der Informationsgesellschaft [...] Markt und Gesellschaft [...] nicht mehr nur von materiellen, sondern zunehmend auch von immateriellen Werten“ bestimmt werden. Hieraus folgt für ihn die verstärkte Anwendung des Immaterialgüterrechts, das allerdings „nur äußerst unzureichend“ auf diese Herausforderung „vorbereitet“ ist (S. 102 f.). Ulrich Sarcinelli rundet den Abschnitt mit einem kenntnisreichen Aufsatz zu „Kommunikation und Partizipation in einer veränderten Legitimationsarchitektur“ ab.

Im zweiten Schwerpunkt stehen konkrete Entwicklungen in Deutschland und den anderen westlichen Demokratien im Mittelpunkt. Alma Kolleck macht hier mit einer abbildungslastigen Abhandlung zu „Kollektive[r] Meinungsbildung Online. Der Einfluss von Webseiten auf politische Online-Diskussionen“ den Anfang. Saskia Richter greift mit dem Thema „Politische Partizipation und soziale Bewegung in sich wandelnden Gesellschaften. Von Bürgerinitiativen zu Online-Netzwerken“ einen Gedanken auf, der für die weitere Entwicklung von Partizipation und Engagement in der Gesellschaft des 21. Jahrhunderts von besonderer Bedeutung ist. Die Beiträge von Florian Hartleb zu „Occupy. Globalisierter globalisierungskritischer Protest als Wellenbewegung“ und Stephan Klecha zu „Politik 2.0 - Die Piratenpartei“ bergen zu den bekannten Arbeiten der Autoren wenig Neuigkeitswert.

Im abschließenden dritten Abschnitt steht die Frage im Mittelpunkt, ob das Internet eine Herausforderung für Autokratien ist oder deren Machterhalt dient. Thomas Demmelhuber präsentiert mit seinen Ausführungen zu „Neue Medien, Protest und politische Veränderung im Nahen Osten: Überlegungen am Beispiel des Arabischen Frühlings“ gewissermaßen „die Gefahr“ und Nele Nosselt mit „Das Internet in China: Public Sphere oder autokratisches Kontrollinstrument?“ den Machterhalt eines Regimes durch gezielten Einsatz der neuen Möglichkeiten.

Die Leitfrage nach Einfluss und Bedeutung des Internets für die Demokratie stellt jeder Beitrag seinem Thema entsprechend. Als Resümee ist festzuhalten, dass die „netzrealistische“ Erschließung einen skeptischen Ton hat und einige aktuelle Entwicklungen mit Sorge betrachtet. So klingt das folgende Diktum in zahlreichen Artikeln an: „Das Internet als solches führt nicht zu einer stärkeren Politisierung der Gesellschaft. Es ist keine ,liberation technology“, sondern ein neutrales Medium“ (Nele Nosselt, S. 274). In Bezug auf das Wechselspiel von „Bereicherung und Stress“ kann gegenwärtig „skeptische Zuversicht“ signalisiert werden (Ulrich Sarcinelli, S. 122).

Als Fazit ist festzuhalten, dass der Band im Ganzen gut strukturiert ist. Er vereint Überblicksartikel zu ideengeschichtlichen Begrifflichkeiten mit konkreten Fragestellungen zu 
unmittelbaren Auswirkungen der Digitalen Revolution auf die Demokratie im 21. Jahrhundert. Formal finden sich einige kleinere Nachlässigkeiten bei der Edition, die den Lesefluss aber nicht stören. Die Aktualität des Themas, die breite Ausrichtung und die Tiefe der Beiträge macht den Tagungsband für eine große Zielgruppe lesenswert.

Christian Nestler

\section{Extremismusforschung: aktuelle Einführung und langfristige Trends in gewohnter Qualität}

Backes, Uwe, Alexander Gallus und Eckhard Jesse (Hrsg.): Jahrbuch Extremismus \& Demokratie (E \& D), 24. Jahrgang 2012, Nomos Verlagsgesellschaft, Baden-Baden 2012, 534 Seiten, $€ 54,-$

Hirscher, Gerhard und Eckhard Jesse (Hrsg.): Extremismus in Deutschland. Schwerpunkte, Vergleiche, Perspektiven (Reihe „Extremismus und Demokratie“, Band 26), Nomos Verlagsgesellschaft, Baden-Baden 2013, 608 Seiten, € 89,-.

In mittlerweile eingespielter Zusammenarbeit dreier Herausgeber entstanden, teilt sich der 24. Band des Jahrbuchs „E \& D“ in gewohnter Weise in die Abschnitte „Analysen“ (S. 13 - 101), „Daten, Dokumente, Dossiers“ (S. 105 - 261), „Literaturteil“ (S. 265 - 503) und Anhänge (S. 504 - 534). Bereits in den "Analysen“ kommt in exemplarischer Form die Problemzugangsweise der Herausgeber zum Ausdruck: Beiträge zu Demokratietheorie und Extremismus mit Äquidistanz nach links und rechts liefern einen Ordnungsrahmen, komplementäre Einzeluntersuchungen zu Detailaspekten von Linksterrorismus und historischem Rechtsextremismus vermitteln Einsichten in langfristige Trends, aktuelle Entwicklungen und zeitgeschichtliche Abläufe.

Extreme Fälle eines Wechsels aus der bürgerlichen Gesellschaft in den gewaltsamen Kampf gegen sie unter Abbruch einer hoffnungsvoll begonnenen akademischen Karriere zeichnet Alexander Gallus anhand von Akten aus der Studienstiftung des Deutschen Volkes über die ehemaligen Stipendiatinnen und Stipendiaten Ulrike Meinhoff, Horst Mahler, Gudrun Ensslin und Bernhard Vesper nach (S. 13 - 29). Die Fassungslosigkeit der Betreuer über Lebensweg und Ende der Geförderten werden vor allem durch Gallus 'Verzicht auf retrospektive Interpretationen plausibel nachvollziehbar.

Zeitgeschichtlichen Abläufen um die erfolglosen Rückkehrversuche des im Gegensatz zu seinem Bruder rechtzeitig aus Deutschland geflohenen „Alten Kämpfers“ der NSDAP Otto Straßer widmet sich Herbert Elzer (S. 72 - 101). Die auf Archivmaterialien gestützte Untersuchung fördert diverse Winkelzüge, Doppelbödigkeiten und Unaufrichtigkeiten in einem nach dem Zweiten Weltkrieg nicht mehr geschichtsmächtig gewordenen Zweig autoritären politischen Denkens zu Tage. Neben vergessenen finden sich teilweise auch prominente Namen, wobei der Autor aus Paul Freiherr von Schönaich-Carolath (S. 81) wenige Seiten später einen Prinzen macht (S. 88).

In übersichtlich-knapper Form ordnet und referiert Manfred G. Schmidt gekonnt Demokratietheorien unterschiedlicher Provenienz und mit divergierenden Prognosen (S. 30 